\title{
Fast Slew Maneuvers for the High-Torque-Wheels BIROS Satellite*
}

\author{
Paul ACQuATElla B. ${ }^{\dagger}$
}

\author{
DLR, German Aerospace Center, Institute of System Dynamics and Control, D-82234 Oberpfaffenhofen, Germany
}

\begin{abstract}
The satellite platform BIROS is the second technology demonstrator of DLR's 'FireBIRD' space mission aiming to provide infrared remote sensing for early fire detection. Among several mission goals and scientific experiments, to demonstrate a high-agility attitude control system, the platform is actuated with an extra array of three orthogonal 'HighTorque-Wheels.' However, to enable agile reorientation, a challenge arises from the fact that time-optimal slew maneuvers are, in general, not of the Euler-axis rotation type; especially whenever the actuators are constrained independently. Moreover, BIROS' on-board computer can only accommodate rotational acceleration commands twice per second. The objective is therefore to find a methodology to design fast slew maneuvers while considering a highly dynamic plant commanded by piecewise-constant sampled-time control inputs. This is achieved by considering a comprehensive analytical nonlinear model for spacecraft equipped with reaction wheels and transcribing a time-optimal control problem formulation into a multi-criteria optimization problem. Solutions are found with a direct approach using the trajectory optimization package 'trajOpt' of DLR-SR's optimization tool, Multi-Objective Parameter Synthesis (MOPS). Results based on numerical simulations are presented to illustrate this method.
\end{abstract}

Key Words: Attitude Control System, Slew Maneuver Optimization, Time-Optimal Control, Sampled-Time Control

\section{Introduction}

The satellite platform Bi-spectral InfraRed Optical System (BIROS), ${ }^{1)}$ successfully launched into space on 22 June 2016 at 05:55 CEST, is the second technology demonstrator along with the TET-1 satellite of the DLR R\&D 'FireBIRD' ${ }^{2)}$ space mission aiming to provide infrared (IR) remote sensing for early fire detection (forest fires, volcanic activity, gas flares and industrial hotspots), see Fig. 1. These small satellites are extensions and largely based on the flight-proven Bispectral Infra-Red Detection (BIRD) $)^{3,4)}$ satellite bus launched in 2001 .

Among several mission goals and scientific experiments, the platform is actuated with an extra array of three orthogonal 'High-Torque-Wheels' (HTW) ${ }^{5,6)}$ to demonstrate a highagility attitude control system. Since the fast slew maneuvers are meant to be performed mainly by the HTW array, the satellite platform's main torque actuators, as with TET-1, are four precise 'RW-90' reaction wheels ${ }^{7)}$ in a redundant tetrahedron configuration. Wheel characteristics for both the HTW and the RW-90 are presented in Table 1, while BIROS' reaction wheel array alignment parameters, as described in the kinematics section, are presented in Table 2.

One of the main requirements for the HTW experiment is being able to perform $30 \mathrm{deg} 1$-axis rotations in $10 \mathrm{~s}$ around a principal axis with an inertia of $10 \mathrm{~kg} \cdot \mathrm{m}^{2}$. For three-axis rotations, rotation paths are, in general, not prescribed in the requirements and these rotations are desired to be performed in

(C) 2018 The Japan Society for Aeronautical and Space Sciences

*Presented at the 26th International Symposium on Space Flight Dynamics, June 3-9, 2017, Matsuyama, Japan.

Received 26 June 2017; final revision received 8 November 2017; accepted for publication 20 December 2017.

†Corresponding author, paul.acquatella@dlr.de minimal time. The experiment is originally designed to be implemented in the 'Fast Slew' mode of BIROS' Attitude Control System (ACS). See Löw et al. ${ }^{8)}$ for a detailed description of other (main) modes, which are similar to the ones implemented for the TET-1 satellite ${ }^{9,10)}$ of the FireBIRD constellation.

However, for agile reorientation, a challenge arises from the fact that time-optimal slew maneuvers are, in general, not of the Euler-axis rotation ${ }^{11,12)}$ type; especially whenever the actuators are constrained independently, ${ }^{13)}$ as it will be in this case. Moreover, the BIROS on-board computer (OBC) can only accommodate rotational acceleration commands twice per second, which means that these must be piecewiseconstant sampled-time control inputs.

The topic of optimal spacecraft rotational maneuvers is quite extensive ${ }^{14)}$ and has been studied for many decades. Earlier works ${ }^{15,16)}$ considered numerical approaches and quasi-closed-form solutions to reorientation problems, while only recently new results have been found for minimum-time and time-optimal reorientation maneuvers ${ }^{13,17-19)}$ for more generic configurations. Some of these results have been experimentally validated for imaging satellites in-orbit. ${ }^{20)}$ Time-optimal reorientation solutions for rigid bodies have also been found using a geometric mechanics approach ${ }^{21,22)}$ together with indirect optimization. However, most of the work reported in literature does not consider time-optimal control solutions of spacecraft equipped with reaction wheels driven by independently constrained piecewise-constant sampled-time control inputs.

This motivates the objective of this paper, which is to find a methodology to design fast slew maneuvers for the BIROS HTW experiment while considering a highly dynamic plant commanded by piecewise-constant sampled-time control inputs. The offline solutions considered in this paper are mainly 


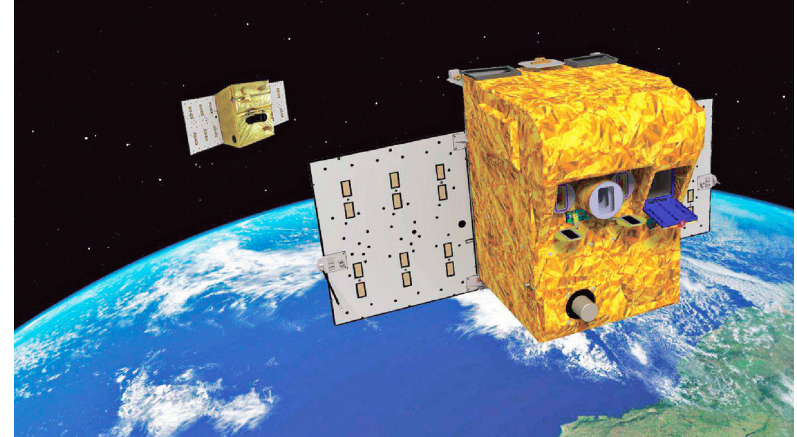

Fig. 1. FireBIRD — a satellite duo for fire detection. BIROS (front), TET-1 (back). Credit: DLR, CC-BY 3.0.

Table 1. Wheel characteristics..$^{6,7,9,10)}$

\begin{tabular}{llll}
\hline Performance & RW-90 & & HTW \\
\cline { 1 - 1 } \cline { 4 - 4 } Nominal speed [rpm] & 6000 & 1825 \\
Max. speed [rpm] & 7800 & 3000 \\
Nominal torque [Nm] & 0.015 & 0.21 \\
Max. torque [Nm] & 0.021 & 0.23 \\
Nominal ang. momentum [Nms] & 0.2639 & 0.9556 \\
Max. ang. momentum [Nms] & 0.3431 & 1.5708 \\
\cline { 1 - 1 } Mechanics & & \\
\cline { 1 - 1 } Number of wheel units & 4 & 3 \\
Moment of inertia $\left[\mathrm{kg} \cdot \mathrm{m}^{2}\right]$ & $4.2 \times 10^{-4}$ & $5 \times 10^{-3}$ \\
\hline
\end{tabular}

Table 2. BIROS' reaction wheel array alignment parameters.

\begin{tabular}{|c|c|}
\hline Alignment vector & Value \\
\hline$a_{1}(\mathrm{HTW})$ & $\left(\begin{array}{lll}1 & 0 & 0\end{array}\right)^{\mathrm{T}}$ \\
\hline $\boldsymbol{a}_{2}$ (HTW) & $\left(\begin{array}{lll}0 & 1 & 0\end{array}\right)^{\mathrm{T}}$ \\
\hline$a_{3}$ (HTW) & $\left(\begin{array}{lll}0 & 0 & 1\end{array}\right)^{\mathrm{T}}$ \\
\hline $\boldsymbol{a}_{4}(\mathrm{RW}-90)$ & $\left(\begin{array}{lll}0 & -1 & 0\end{array}\right)^{\mathrm{T}}$ \\
\hline $\boldsymbol{a}_{5}(\mathrm{RW}-90)$ & $\left(\begin{array}{lll}\frac{1}{2} \sqrt{3} \sin \theta & \cos \theta & -\frac{1}{2} \sin \theta\end{array}\right)^{\mathrm{T}}$ \\
\hline $\boldsymbol{a}_{6}(\mathrm{RW}-90)$ & $\left(\begin{array}{lll}-\frac{1}{2} \sqrt{3} \sin \theta & \cos \theta & -\frac{1}{2} \sin \theta\end{array}\right)$ \\
\hline $\boldsymbol{a}_{7}(\mathrm{RW}-90)$ & $\left(\begin{array}{lll}0 & \cos \theta & \sin \theta\end{array}\right)^{\mathrm{T}}$ \\
\hline \multicolumn{2}{|l|}{ Alignment angle } \\
\hline$\theta[\mathrm{rad}]$ & $\arccos (-1 / 3)$ \\
\hline
\end{tabular}

oriented to rest-to-rest maneuvers and will be implemented as sampled-input feedforward commands in combination with error feedback control in a two-degrees-of-freedom control system architecture.

This is achieved by 1) considering a comprehensive analytical nonlinear model for spacecraft equipped with reaction wheels; 2) considering the outer-loop control as the feedforward commands designed here; 3 ) transcribing a time-optimal control problem formulation into a direct approach involving a multi-criteria optimization problem considering inequality and equality constraints; and 4) solving the transcribed problem directly using the trajectory optimization package 'trajOpt' of DLR-SR's optimization tool, Multi-Objective Parameter Synthesis (MOPS). To obtain the desired piecewise-constant sampled-time inputs, the methodology proposed follows a sequential three-step procedure. Finally, numerical simulations of the procedure steps proposed are presented.

\section{Modeling of Spacecraft with Reaction Wheels}

In this section a comprehensive nonlinear rotational dynamics model for spacecraft is described. The model includes a generic set of reaction wheels in arbitrary configuration which are driven by exogenous inputs provided by each wheel's powertrain.

\subsection{Kinematics}

Consider, first, an array consisting of $n$ reaction wheels. Introducing unit vectors $\boldsymbol{a}_{i}$, which give the orientation of the spin-axis of each reaction wheel with respect to the spacecraft coordinate system collected in the configuration or alignment matrix

$$
A=\left[\boldsymbol{a}_{1}\left|\boldsymbol{a}_{2}\right| \cdots \mid \boldsymbol{a}_{n}\right],
$$

then each $\boldsymbol{a}_{i}$ can define the $i$-th reaction wheel or 'actuator' frame by taking $\boldsymbol{a}_{i}$ as the first axis and making the remaining axes constitute an orthogonal frame. The kinematics of the $i$-th reaction wheel with respect to its corresponding actuator frame in terms of its spin-axis angle $\Phi_{w, i}$ and angular velocity $\Omega_{w, i}$ is simply given by

$$
\dot{\Phi}_{w, i}=\Omega_{w, i}, \quad i=1, \ldots, n .
$$

Next, consider the spacecraft equipped with the $n$ reaction wheels just introduced. Rotation matrices $\boldsymbol{R} \in \mathcal{S O}(3)$, representing a linear transformation of vectors in body-fixed or 'hub' frame into the inertial frame, are preferred as the attitude parameterization since they are both global and unique, ${ }^{23)}$ where the configuration space or manifold of rotation matrices ${ }^{21)}$ is given by the special orthogonal group $\mathcal{S O}(3)$ with the conditions

$$
\mathcal{S O}(3)=\left\{\boldsymbol{R} \in \mathcal{R}^{3 \times 3} \mid \boldsymbol{R}^{\mathrm{T}} \boldsymbol{R}=\mathcal{I}_{3 \times 3}, \operatorname{det}[\boldsymbol{R}]=1\right\} .
$$

In that sense, the kinematics of the full spacecraft with respect to the inertial frame, and in terms of its rotation matrix $\boldsymbol{R}$ and angular velocity $\omega \in \mathcal{R}^{3}$, is given by

$$
\dot{\boldsymbol{R}}=\boldsymbol{R} \cdot S(\boldsymbol{\omega}) .
$$

The skew map $S(\cdot): \mathcal{R}^{3} \mapsto \mathfrak{s o ( 3 )}$ is a linear isomorphism between $\mathcal{R}^{3}$ and the Lie algebra $\mathfrak{s o}(3)$, which represents $3 \times 3$ skew-symmetric matrices, and is defined by the condition that $S(x) y=x \times y$ for any $x, y \in \mathcal{R}^{3}$, or algebraically as

$$
S(x)=\left[\begin{array}{rcc}
0 & -x_{3} & x_{2} \\
x_{3} & 0 & -x_{1} \\
-x_{2} & x_{1} & 0
\end{array}\right] .
$$

The inverse of the skew map is denoted by the vee map $\vee: \mathfrak{s o}(3) \mapsto \mathcal{R}^{3}$.

\subsection{Dynamics}

Following the derivations in Karpenko et al., ${ }^{20)}$ a rotational dynamics model is obtained as follows. First, consider the angular momentum of the spacecraft equipped with the reaction wheel array in question

$$
H=I \omega+h
$$


where, expressed in a body-fixed frame, $\boldsymbol{H} \in \mathcal{R}^{3}$ is the total angular momentum of the system, $\boldsymbol{I} \in \mathcal{R}^{3 \times 3}$ is the constant inertia matrix of the spacecraft including the reaction wheels, $\omega \in \mathcal{R}^{3}$ is the spacecraft angular velocity, and $\boldsymbol{h} \in \mathcal{R}^{3}$ is the total angular momentum vector associated with the reaction wheel array. The angular momentum $\boldsymbol{h}$ can be expressed from individual actuator frames to the body-fixed frame as

$$
\boldsymbol{h}=\sum_{i=1}^{n} \boldsymbol{a}_{i} \boldsymbol{h}_{w, i}=A \boldsymbol{I}_{\boldsymbol{w}} \boldsymbol{\Omega},
$$

where $\boldsymbol{I}_{\boldsymbol{w}}$ is a diagonal matrix of reaction wheel spin-axis inertia values

$$
\boldsymbol{I}_{\boldsymbol{w}}=\left[\begin{array}{ccc}
\boldsymbol{I}_{w, 1} & \cdots & 0 \\
\vdots & \ddots & \vdots \\
0 & \cdots & \boldsymbol{I}_{w, n}
\end{array}\right],
$$

and $\boldsymbol{\Omega}$ is the inertial angular rate of the reaction wheel array

$$
\boldsymbol{\Omega}=\Omega_{w}+A^{\mathrm{T}} \boldsymbol{\omega} .
$$

The term $A^{\mathrm{T}} \boldsymbol{\omega}$ is the extra angular motion relative to the spacecraft. Considering the angular momentum associated with the $i$-th reaction wheel in the actuator frame

$$
\boldsymbol{h}_{w, i}=\boldsymbol{I}_{w, i}\left(\Omega_{w, i}+\boldsymbol{a}_{i}^{\mathrm{T}} \boldsymbol{\omega}\right), \quad i=1, \ldots, n,
$$

after which the differential equation describing the reaction wheel dynamics in terms of reaction wheel torques $\tau_{w, i}$ can be obtained

$$
\dot{\Omega}_{w, i}=\boldsymbol{I}_{w, i}^{-1} \tau_{w, i}-\boldsymbol{a}_{i}^{\mathrm{T}} \dot{\boldsymbol{\omega}}, \quad i=1, \ldots, n .
$$

Here, the reaction wheel torques are considered as the exogenous inputs to the system and are provided by the wheel's powertrain.

Because the angular momentum must be conserved in the absence of external perturbations, applying the transport theorem $^{14,20)}$ to Eq. (4), the following relation is obtained

$$
\frac{\mathrm{d}}{\mathrm{d} t} \boldsymbol{H}+\boldsymbol{\omega} \times \boldsymbol{H}=0,
$$

which can be further expanded as

$$
\boldsymbol{I} \dot{\boldsymbol{\omega}}+A \boldsymbol{I}_{w} \dot{\boldsymbol{\Omega}}+\boldsymbol{\omega} \times\left(\boldsymbol{I} \boldsymbol{\omega}+A \boldsymbol{I}_{w} \boldsymbol{\Omega}\right)=0 .
$$

Combining Eqs. (5), (7), and (9), the comprehensive nonlinear model for spacecraft dynamics equipped with reaction wheels $^{20)}$ is given by

$$
\boldsymbol{\Gamma}\left[\begin{array}{c}
\dot{\boldsymbol{\omega}} \\
\dot{\Omega}_{w, 1} \\
\vdots \\
\dot{\Omega}_{w, n}
\end{array}\right]=\left[\begin{array}{c}
-\boldsymbol{\omega} \times\left(\boldsymbol{I} \boldsymbol{\omega}+A \boldsymbol{I}_{w} \Omega_{w}+A \boldsymbol{I}_{\boldsymbol{w}} A^{\mathrm{T}} \boldsymbol{\omega}\right) \\
\tau_{w, 1} \\
\vdots \\
\tau_{w, n}
\end{array}\right]
$$

where

$$
\boldsymbol{\Gamma}=\left[\begin{array}{cccc}
\boldsymbol{I}+A \boldsymbol{I}_{w} A^{\mathrm{T}} & \boldsymbol{a}_{1} \boldsymbol{I}_{w, 1} & \cdots & \boldsymbol{a}_{n} \boldsymbol{I}_{w, n} \\
\boldsymbol{I}_{w, 1} \boldsymbol{a}_{1}^{\mathrm{T}} & \boldsymbol{I}_{w, 1} & \cdots & 0 \\
\vdots & \vdots & \ddots & \vdots \\
\boldsymbol{I}_{w, n} \boldsymbol{a}_{n}^{\mathrm{T}} & 0 & \cdots & \boldsymbol{I}_{w, n}
\end{array}\right]
$$

is an augmented inertia coupling matrix for the full system.

\section{Attitude Control}

\subsection{Reaction wheel inner-loop control}

Each wheel torque $\tau_{w, i}$ consists of a motor-provided torque $\tau_{m, i}$ and an undesired friction torque $\tau_{f, i}$

$$
\tau_{w, i}=\tau_{m, i}+\tau_{f, i}, \quad i=1, \ldots, n,
$$

where the friction torque results from static, viscous, Coulomb, an other nonlinear friction torques related to stiction and to extreme conditions of the space environment. The friction torque is estimated with a simple model as

$\hat{\tau}_{f, i}=M_{\mathrm{vis}} \Omega_{w, i}+M_{\text {Coul }} \operatorname{sign}\left(\Omega_{w, i}\right), \quad i=1, \ldots, n$,

where $M_{\text {vis }}$ and $M_{\text {Coul }}$ are viscous and Coulomb friction parameters, respectively. When no gearboxes are present, and neglecting the dynamics of the DC-motor's electrical current $i_{c}$, the relationship between motor current and motor output can be assumed as

$$
\tau_{m, i}=\eta_{m} K_{m} i_{c},
$$

where $\eta_{m}$ and $K_{m}$ are the motor efficiency and motor constant, respectively. However, to compensate for undesired friction torques $\tau_{f, i}$, a reaction-wheel inner-loop controller embedded in the actuator and operating at a sampling rate of $100 \mathrm{~Hz}$ is designed to compensate the effect of undesired and estimated wheel friction torques as a nonlinear function

$$
\tau_{m, i}=f_{w}\left(\tau_{w, i_{c m d}}, \hat{\tau}_{f, i}, \hat{\Omega}_{w, i}, \hat{\Phi}_{w, i}\right)
$$

which tracks the wheel-torque reference command $\tau_{w, i_{c m}}$ with the estimated quantities for friction, wheel velocity, and wheel angle. The torque reference command can be related to a desired wheel acceleration whenever wheel-rate control is required by

$$
\tau_{w, i_{c m d}}=\hat{\boldsymbol{I}}_{w, i} \dot{\Omega}_{w, i_{d e s}}
$$

where $\hat{\boldsymbol{I}}_{w, i}$ is an estimate of the $i$-th wheel inertia. Finally, the $i$-terms $\tau_{w, i_{c m d}}$ are collected on a single vector as

$$
\boldsymbol{u}_{w}=\left[\begin{array}{c}
\tau_{w, 1} \\
\vdots \\
\tau_{w, n}
\end{array}\right]_{c m d} .
$$

As mentioned in the introduction, BIROS' OBC can only accommodate commands at a sampling rate of $2 \mathrm{~Hz}$; therefore, to perform fast slew maneuvers, an outer-loop controller is designed such that it commands the wheel torques in $k$-sampled times as $\boldsymbol{u}_{w}=\boldsymbol{u}_{w}(k)$ for $k \in\{0, \ldots, N\}$, where $N$ represents the maneuver's final time sample.

\subsection{Attitude and rate outer-loop control}

Analogous to Eq. (3), a smooth attitude command $\boldsymbol{R}_{d} \in \mathcal{S O}(3)$ satisfying

$$
\dot{\boldsymbol{R}}_{d}=\boldsymbol{R}_{d} \cdot S\left(\omega_{d}\right)
$$

is considered, where $\omega_{d}$ is the desired angular velocity assumed to be uniformly bounded. Lee ${ }^{22}$ showed that careful 
selection of an attitude error function can guarantee good tracking performance of nontrivial slew maneuvers involving large initial attitude errors. This is because the magnitude of an attitude error vector should be proportional to the rotation about the Euler-axis between the current and desired attitude. In this sense, an attitude error function $\Psi: \mathcal{S O}(3) \times$ $\mathcal{S O}(3) \mapsto \mathcal{R}$, as in Goodarzi et al., ${ }^{24)}$ is selected such that

$$
\Psi\left(\boldsymbol{R}, \boldsymbol{R}_{d}\right)=\frac{1}{2} \operatorname{tr}\left(I-\boldsymbol{R}_{d}^{\mathrm{T}} \boldsymbol{R}\right)
$$

where $\operatorname{tr}(\cdot)$ denotes the trace of a square matrix. With this choice, an attitude error vector $\boldsymbol{e}_{R} \in \mathcal{R}^{3}$ and angular velocity error vector $\boldsymbol{e}_{\omega} \in \mathcal{R}^{3}$ can be defined as

$$
\begin{aligned}
\boldsymbol{e}_{R} & =\frac{1}{2}\left(\boldsymbol{R}_{d}^{\mathrm{T}} \boldsymbol{R}-\boldsymbol{R}^{\mathrm{T}} \boldsymbol{R}_{d}\right)^{\vee}, \\
\boldsymbol{e}_{\omega} & =\boldsymbol{\omega}-\boldsymbol{R}^{\mathrm{T}} \boldsymbol{R}_{d} \boldsymbol{\omega}_{d},
\end{aligned}
$$

recalling that $\vee$ denotes the vee map as defined in Section 2. Note that the magnitude of the dimensionless attitude error vector is bounded ${ }^{22)}$ as $0 \leq\left|\boldsymbol{e}_{R}\right| \leq 1$. The sampled-time tracking error state $\boldsymbol{x}_{\boldsymbol{e}}(k) \in \mathcal{R}^{6}$ is defined as

$$
\boldsymbol{x}_{\boldsymbol{e}}(k)=\left[\begin{array}{c}
\boldsymbol{e}_{R}(k) \\
\boldsymbol{e}_{\omega}(k)
\end{array}\right]
$$

and the objective is therefore to design an attitude control law having $\boldsymbol{x}_{\boldsymbol{e}} \rightarrow 0$ as $k \rightarrow N$. This means that $\boldsymbol{x}_{\boldsymbol{e}}=0$ if and only if $\boldsymbol{R}=\boldsymbol{R}_{d}$ and therefore $\boldsymbol{\omega}=\boldsymbol{R}^{\mathrm{T}} \boldsymbol{R}_{d} \boldsymbol{\omega}_{d}=\boldsymbol{\omega}_{d}$. Sampledtime nonlinear attitude control is given by a combination of feedback and feedforward control laws

$$
\boldsymbol{u}_{w}(k)=\boldsymbol{u}_{F B}(k)+\boldsymbol{u}_{F F}(k),
$$

where $\boldsymbol{u}_{F B}$ can be the discrete version of the geometric PID attitude controller proposed in Goodarzi et al. ${ }^{24)}$ without the feedforward terms. In the next section, the feedforward commands $\boldsymbol{u}_{F F}(k)$ obtained as solutions of time-optimal control problems are described.

\section{Optimal Guidance}

In this section, a methodology is presented for the generation of offline fast slew maneuvers as solutions of timeoptimal control problems. The solutions serve as the basis for the attitude control system where they will be implemented as the feedforward control commands $\boldsymbol{u}_{F F}(k)$ in sampled-time.

4.1. Time-optimal slew maneuver problem formulation

The objective of time-optimal slew maneuver problems ${ }^{20,21)}$ consists on finding optimal wheel-motor torque commands $\tau_{w, i}(i=1, \ldots, n)$ that transfer any given initial attitude $\boldsymbol{R}\left(t_{0}\right)$, angular velocity $\boldsymbol{\omega}\left(t_{0}\right)$, and wheel speed $\Omega_{w}\left(t_{0}\right)$ of the rigid body to a desired final attitude $\boldsymbol{R}\left(t_{f}\right)$, angular velocity $\omega\left(t_{f}\right)$, and wheel speed $\Omega_{w}\left(t_{f}\right)$ within a minimal time $t_{f}$. Such time-optimal maneuvers can be mathematically formulated as the following optimization problem

$$
\underset{\tau_{w, i},(i=1, \ldots, n)}{\operatorname{minimize}}\left\{J=\int_{t_{0}}^{t_{f}} 1 \mathrm{~d} t\right\},
$$

subject to the dynamic Eqs. (3) and (10), $\forall t \in\left[t_{0}, t_{f}\right]$,

$$
\begin{aligned}
& \text { such that } \boldsymbol{R}\left(t_{0}\right)=\boldsymbol{R}_{0} \text {, } \\
& \boldsymbol{R}\left(t_{f}\right)=\boldsymbol{R}_{f}, \\
& \boldsymbol{\omega}\left(t_{0}\right)=\omega_{0}, \\
& \omega\left(t_{f}\right)=\omega_{f}, \\
& \Omega_{w}\left(t_{0}\right)=\Omega_{w 0}, \\
& \Omega_{w}\left(t_{f}\right)=\Omega_{w f},
\end{aligned}
$$

$$
\left\|\tau_{w, i}(t)\right\| \leq \tau_{w, i_{\max }}, \quad(i=1, \ldots, n), \quad \forall t \in\left[t_{0}, t_{f}\right] .
$$

Without loss of generality, only rest-to-rest maneuvers are considered in this work, where initial and final angular velocities are directly imposed to be zero:

$$
\omega\left(t_{0}\right)=\omega\left(t_{f}\right)=\left(\begin{array}{lll}
0 & 0 & 0
\end{array}\right)^{\mathrm{T}} \mathrm{rad} / \mathrm{s} .
$$

Moreover, in the remainder of this paper, initial HTW speeds are also considered to be zero $\Omega_{w, i}\left(t_{0}\right)=0, i=1,2,3$, and their final wheel speed is set to be free. The remaining RW-90 wheels, $i=4, \ldots, 7$, are set-point regulated according to their initial values with a simple proportional control law as

$$
\dot{\Omega}_{w, i}=-k_{p}\left[\Omega_{w, i}-\Omega_{w, i}\left(t_{0}\right)\right], \quad k_{p}=1 \times 10^{-4}
$$

giving rise to non-cooperating angular momentum for the slew maneuvers. Although it has already been mentioned that time-optimal maneuvers are, in general, not Euler-axis rotations whenever the actuators can be saturated independently, it is not straightforward to conclude whether or not a local solution of this problem corresponds to a global solution. 4.2. Transcription of the time-optimal slew maneuver problem formulation into a direct approach

Because the problem formulation of time-optimal slew maneuvers does not involve a prescribed path to be followed a-priori, it can be considered a trajectory optimization problem that minimizes the total maneuver time according to the set of constraints presented.

In this sense, the trajectory optimization problem consists of transcribing the time-optimal control problem into a constrained parameter optimization problem and solving it with a direct approach using DLR's Trajectory Optimization Package ${ }^{25)}$ 'trajOpt,' that is included in the MOPS software environment ${ }^{26-28)}$ and implemented in MATLAB ${ }^{29)}$ which solves multi-objective design problems that are mapped to weighted min-max optimization problems. MOPS is a versatile tool widely used in the aeronautical community ${ }^{26-28,30-34)}$ to support many aspects of general control design processes, such as multi-model and multi-case design problems, robust tuning via Monte-Carlo simulations, control law robustness assessment, worst-case analysis, and parameter estimation. A key advantage of using the trajectory optimization package trajOpt/MOPS, originally designed to solve hybrid multiphase trajectory optimization problems for launch vehicles, is that boundary conditions at the beginning and end of each phase of the desired maneuvers are considered in an efficient way. 
Transcription of the original constrained minimization problem into a direct approach consists on defining the original $k$ design objectives mathematically as positive criteria $c_{k}$ to be minimized against demanded values $d_{k}$, and considering the following min-max multi-criteria optimization problem, which is the MOPS synthesis ${ }^{27,28,31)}$ formula

$$
\min _{\mathcal{T}}\left\{\max _{k \in \mathcal{S}_{m}}\left\{\frac{c_{k}(\mathcal{T})}{d_{k}}\right\}\right\},
$$

subject to $c_{k}(\mathcal{T})=d_{k}, \quad k \in \mathcal{S}_{\text {eq }}$,

$$
\begin{array}{r}
c_{k}(\mathcal{T}) \leq d_{k}, \quad k \in \mathcal{S}_{\text {ineq }}, \\
\text { with } \\
\mathcal{T}_{\text {min }, l} \leq \mathcal{T}_{l} \leq \mathcal{T}_{\text {max }, l}, \quad \forall t \in\left[0, t_{f}\right] .
\end{array}
$$

Here, $\mathcal{S}_{m}$ is the index set of criteria to be minimized, $\mathcal{S}_{\text {eq }}$ is the index set of equality constraints and $\mathcal{S}_{\text {ineq }}$ is the index set of inequality constraints; $\mathcal{T}$ is the vector containing the tuning parameters $\mathcal{T}_{l}$ to be optimized, which lies in between upper and lower bounds $\mathcal{T}_{\min , l}$ and $\mathcal{T}_{\text {max }, l}$, respectively; $c_{k}\left(k \in \mathcal{S}_{m}\right)$ is the $k$-th normalized criterion; $d_{k}\left(k \in \mathcal{S}_{m}\right)$ is the corresponding demand value which serves as a criterion weight; $c_{k}\left(k \in \mathcal{S}_{\text {eq }}, \mathcal{S}_{\text {ineq }}\right)$ is normalized criteria used as equality or inequality constraints, respectively; and lastly, $d_{k}\left(k \in \mathcal{S}_{\text {eq }}, \mathcal{S}_{\text {ineq }}\right)$ is the corresponding demand value. Finally, the newly formulated multi-criteria optimization problem in Eq. (26) can be solved using standard nonlinear programming (NLP) methods for the objective function with equality and inequality constraints.

\subsection{Methodology to obtain piecewise-constant sampled-time optimal maneuvers}

For the main objective of this paper, which is to design fast slew rest-to-rest maneuvers for BIROS' HTW experiments with piecewise-constant sampled-time inputs as feedforward control commands, a methodology is presented. It consists of an iterative procedure that finds solutions to three consecutive problems that are solved using the direct approach previously outlined. Table 3 presents the criteria $c_{k}$, demands $d_{k}$, and tuners $\mathcal{T}$ used for designing the maneuvers considered in this iterative procedure. Note that criteria scaling ${ }^{34)}$ can be performed by dividing each criterion by the value demanded $\hat{c}_{k}(\mathcal{T})=c_{k}(\mathcal{T}) / d_{k}$, and therefore, the value demanded for minimizing the slew time is set to $d_{1}=1 \mathrm{~s}$. The three consecutive problems to be solved are described in detail as follows.

Problem I First, the criteria $c_{1}-c_{3}$ are used together with their demands $d_{1}-d_{3}$ and tuners $\mathcal{T}_{1}$ and $\mathcal{T}_{2}$ to obtain a candidate minimum maneuver time $t_{f}$. Here, the input control commands are interpolated with piecewise cubic Hermite interpolating polynomials ('pchip') available in the trajOpt package in order to obtain a smooth solution for these inputs. The optimal slew time $t_{f}$ is approximated towards a new demanded fixed-time $t_{f}^{*}$, which must be a multiple of the desired frequency of $2 \mathrm{~Hz}$. Additionally, the optimal control inputs are re-sampled at this frequency since they are meant to be used as initial guesses for the subsequent optimi-

\begin{tabular}{|c|c|c|}
\hline \multicolumn{3}{|c|}{ Criteria $c_{k}$} \\
\hline No. & Criteria specification & Description \\
\hline$c_{1}$ & Minimum slew time $t_{f}$ & $t_{f}$ \\
\hline$c_{2}$ & Final attitude error $\boldsymbol{e}_{R}\left(t_{f}\right)$ & $\left|\boldsymbol{e}_{R}\left(t_{f}\right)\right|$ \\
\hline$c_{3}$ & Final angular velocity error $\boldsymbol{e}_{\omega}\left(t_{f}\right)$ & $\left|\boldsymbol{e}_{\omega}\left(t_{f}\right)\right|$ \\
\hline \multicolumn{3}{|c|}{ Demands $d_{k}$} \\
\hline No. & Demands & Value \\
\hline$d_{1}$ & Slew time $t_{f}$ & $1[\mathrm{~s}]$ \\
\hline$d_{2}$ & Final attitude error $\boldsymbol{e}_{R}\left(t_{f}\right)$ & $\leq 1 \times 10^{-7}[-]$ \\
\hline$d_{3}$ & Final angular velocity error $\boldsymbol{e}_{\omega}\left(t_{f}\right)$ & $\leq 1 \times 10^{-5}[\mathrm{rad} / \mathrm{s}]$ \\
\hline \multicolumn{3}{|c|}{ Tuners $\mathcal{T}_{k}$} \\
\hline No. & Tuner & Description \\
\hline $\mathcal{T}_{1}$ & Slew time $t_{f}$ & $t_{f}$ \\
\hline $\mathcal{T}_{2}$ & HTW torque commands & $\tau_{w, i}(i=1,2,3)$ \\
\hline
\end{tabular}
zation problem. With the solution of this problem, an insight
Table 3. Design criteria $c_{k}$, demands $d_{k}$, and tuners $\mathcal{T}_{k}$ used for the design of fast slew maneuvers with trajOpt/MOPS.

can be obtained not only on the minimum time required to complete the maneuver, but also on the maneuver itself since these can be compared, for instance, to Euler-axis rotations which are generally not time-optimal.

Problem II Here, the interest is towards fixed-time solutions for the same problem setup as before, but considering sampled-time control inputs at the sampling rate of $2 \mathrm{~Hz}$. The new demanded fixed-time $t_{f}^{*}$ and the initial guess for the solution are obtained as described in the previous problem. This problem is solved considering criteria $c_{2}-c_{3}$ together with demands $d_{2}-d_{3}$ and tuner $\mathcal{T}_{2}$. In this case, the inputs are obtained as piecewise-linear control commands in order to obtain a sampled-time solution close to the previous one. Once finished, these piecewise-linear solutions are interpolated with a mid-point rule in order to be considered as initial guesses for the next and final optimization problem.

Problem III Here, the criteria $c_{2}-c_{3}$ are again considered together with demands $d_{2}-d_{3}$ and tuner $\mathcal{T}_{2}$. The goal is to find piecewise-constant control inputs for the original problem within the minimum fixed-time $t_{f}^{*}$ approximation obtained before, which represents the final goal of this procedure. The initial guesses obtained from the piecewise-linear inputs of the previous problem are of great help for final optimization since the resulting sampled-time piecewiseconstant control inputs are, in general, already sufficiently close to the optimal desired solution.

Figure 2 presents a diagram of the steps involved in these three consecutive problems. Whenever one of these problems fail to give a feasible solution, a new iteration process is required where the criteria and their demands are re-evaluated. For instance, if no feasible solution for Problem II is found, a good starting point is reconsidering the fixed-time for this problem to be one sample higher, giving an extra control command for the potential new solution. This process is repeated until a satisfactory outcome is achieved.

\section{Simulation}

For numerical simulations using the comprehensive analytical nonlinear model of Section 2, the High-Torque- 
Wheels BIROS satellite is considered with an approximated inertia matrix of

$$
\boldsymbol{I}=\operatorname{diag}\left[\begin{array}{lll}
9 & 6 & 9
\end{array}\right] \mathrm{kg} \cdot \mathrm{m}^{2} .
$$

The time-optimal rest-to-rest maneuver is designed to achieve the desired final attitude described by the $(3-2-1)$

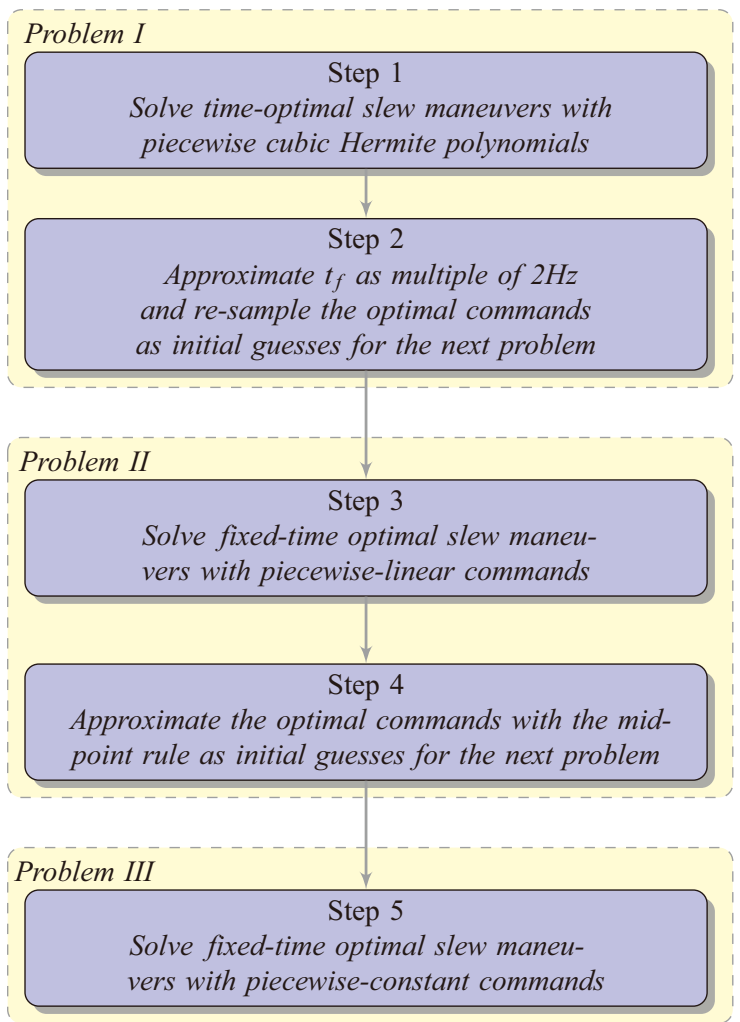

Fig. 2. Diagram of the sequential three-step procedure to obtain fast slew maneuvers with piecewise-constant control commands.
Euler angles $\psi, \theta$, and $\phi$, i.e. $\boldsymbol{R}\left(t_{f}\right)=\boldsymbol{R}\left(\phi\left(t_{f}\right), \theta\left(t_{f}\right), \psi\left(t_{f}\right)\right)$, where $\phi\left(t_{f}\right)=0 \mathrm{deg}, \theta\left(t_{f}\right)=5 \mathrm{deg}$, and $\psi\left(t_{f}\right)=30 \mathrm{deg}$, respectively. This translates into the following initial and final (objective) attitudes in terms of rotation matrices

$$
\boldsymbol{R}\left(t_{0}\right)=\mathcal{I}_{3 \times 3}, \quad \boldsymbol{R}\left(t_{f}\right)=\left[\begin{array}{rrl}
0.8627 & 0.4981 & -0.0872 \\
-0.5000 & 0.8660 & 0 \\
0.0755 & 0.0436 & 0.9962
\end{array}\right] .
$$

It is important to mention that time-dependent attitude paths like Euler-axis rotations or Euler angles are not specified apriori, giving the optimization solver the possibility to find a time optimal path dynamically. The initial HTW wheel speeds are zero since the experiments consider these wheels only for agile reorientation; while the initial RW-90 wheel speeds are set to $\Omega_{w, i}\left(t_{0}\right)=-200 \mathrm{rad} / \mathrm{s}(i=4, \ldots, 7)$ to simulate a realistic scenario of initial angular momentum stored in the platform. The final HTW and RW-90 wheel speeds are set to be free; but actually, the final state of the latter set of wheels depends on the performance of the wheelcontroller in Eq. (25) during the maneuver. Lastly, the nominal values presented in Table 1 are considered as the actuator limits to allow some margin in case the wheels must be saturated by their inner-loop controls.

The simulation results are as follows. Figure 3 presents the torque command solutions using the methodology described in Section 4.3, where the three consecutive optimal control solutions are denoted as $\tau_{w, \mathrm{I}}, \tau_{w, \mathrm{II}}$, and $\tau_{w, \mathrm{III}}$ for each problem I, II, and III, respectively. The solution of problem I gives $t_{f}=9.43 \mathrm{~s}$, so the new demanded fixed-time for problems II and III is set to $t_{f}^{*}=9.5 \mathrm{~s}$ (first multiple of $2 \mathrm{~Hz}$ after $t_{f}$ ). For the optimal control inputs obtained, Fig. 4 presents the simulation results for attitude errors, angular velocities, and reaction wheel speeds, respectively. These results show that the maneuvers are almost identical for the three consec-
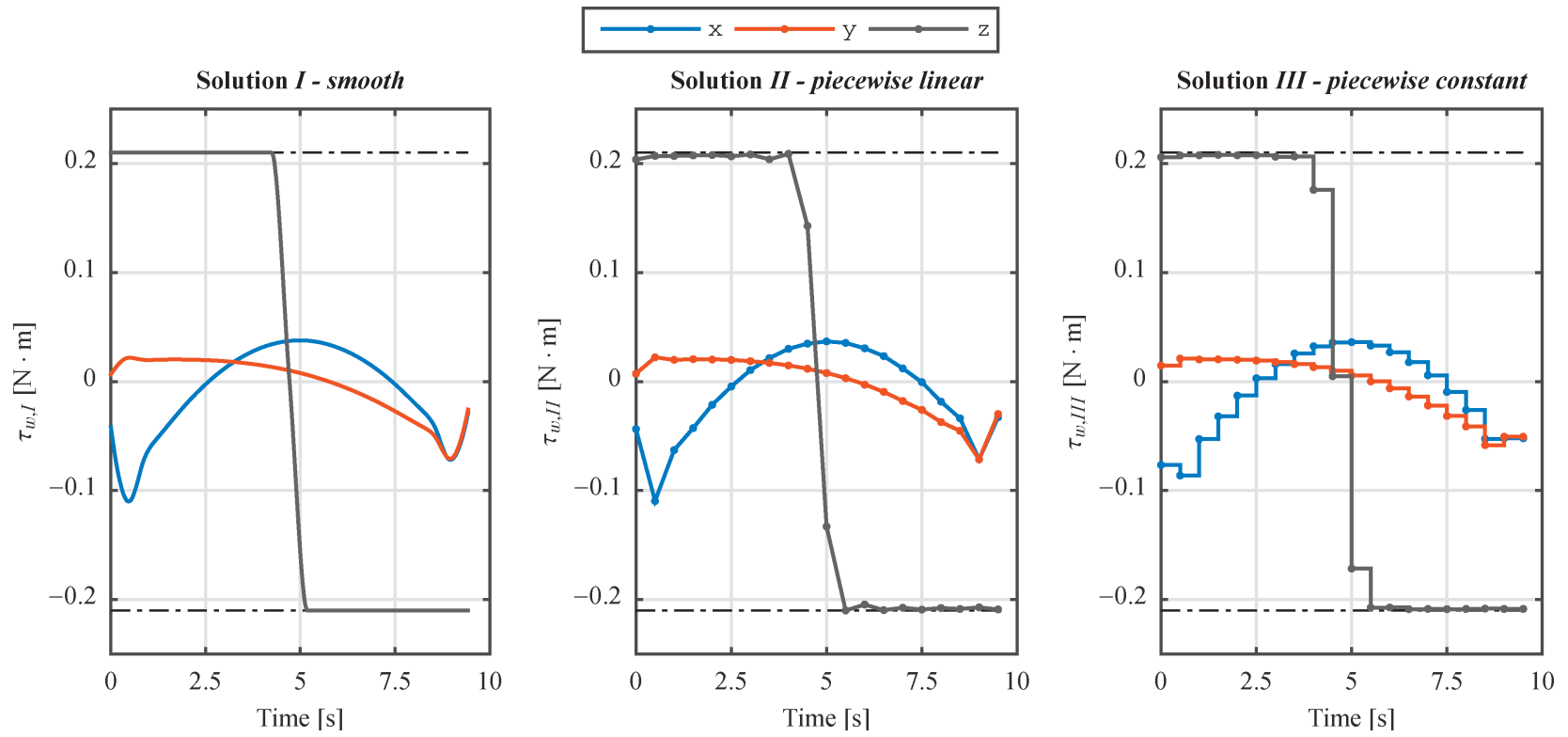

Fig. 3. Torque command results using the sequential methodology to obtain sampled-time fast slew maneuvers; I) first solution finding the minimum time with smooth control inputs; II) second solution with fixed-time and piecewise-linear control inputs; and III) final solution of the original problem with fixedtime and piecewise-constant control inputs. 

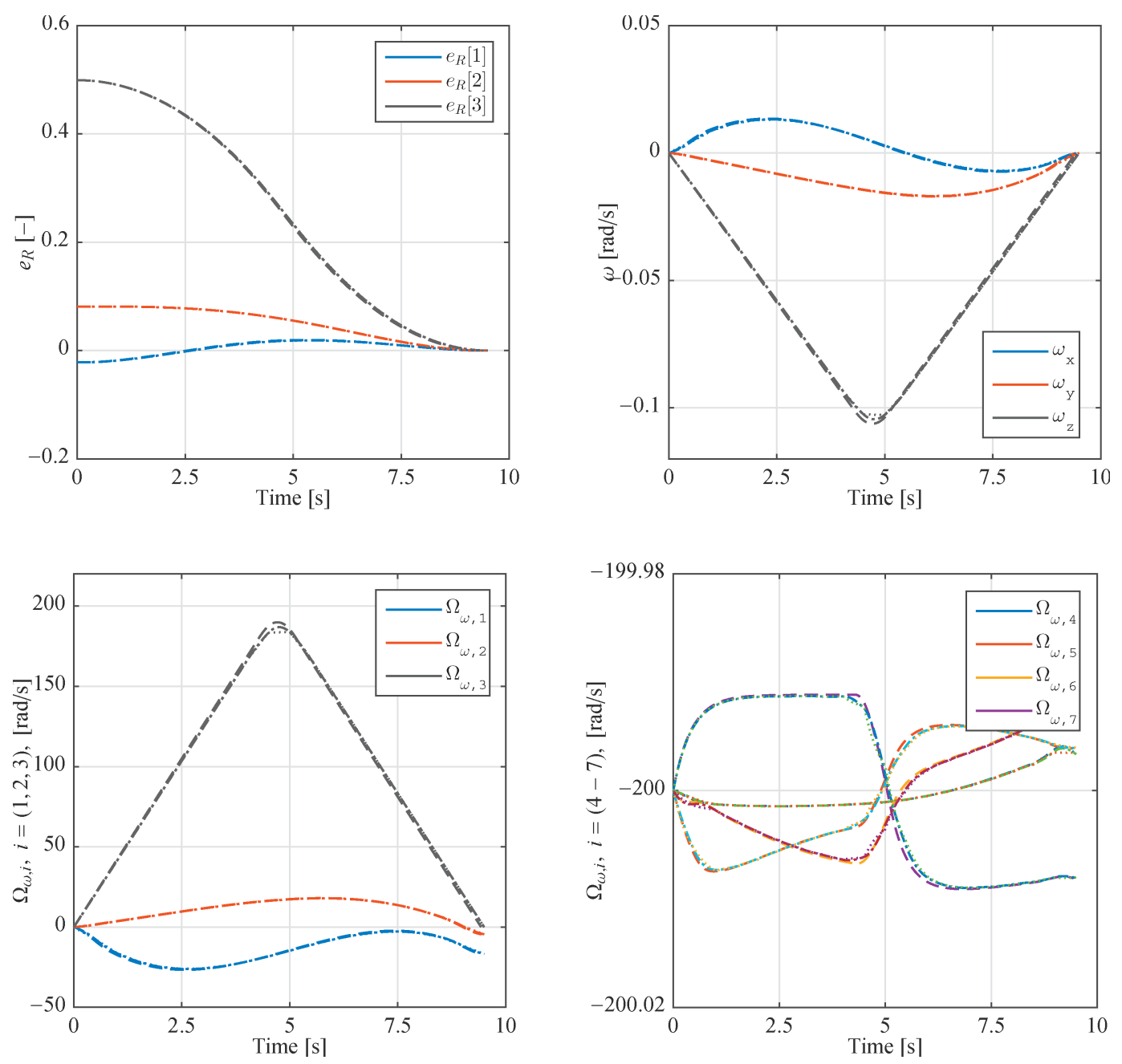

Fig. 4. Simulation results for the attitude error, angular velocity, and reaction wheel speeds, respectively; using the optimal control inputs obtained with solution I (--.--), solution II (…...), and solution III (---).

utive solutions and only differ in the input solutions due to the parameterization of the torque commands. Finding time-optimal maneuvers departing with piecewise-constant control inputs is, in general, a very challenging and time consuming task. Using the three-step methodology presented in this study, the final goal of obtaining fast slew maneuver solutions with piecewise-constant control commands is achieved much more efficiently.

\section{Conclusions and Outlook}

The objective of this paper was to investigate a high-agility attitude control system by finding a methodology to design time-optimal slew maneuvers for BIROS' High-TorqueWheels experiments. This is achieved by considering a comprehensive analytical nonlinear model for spacecraft equipped with reaction wheels and formulating the problem as a constrained nonlinear optimal control problem including both satellite's continuous-time dynamics and piecewiseconstant sampled-time control inputs.

A methodology that utilizes three consecutive multi-criteria optimization problems is proposed to obtain solutions via a direct approach that applies the 'trajOpt' trajectory optimi- zation package of the DLR-SR optimization tool, MultiObjective Parameter Synthesis (MOPS). Results based on numerical simulations performed with the nonlinear spacecraft dynamics model were presented.

Hardware-in-the-loop simulations will be used to validate the attitude control system with a three-axis air-bearing testbed featuring the BIROS engineering model. Once tested, the experiment can be implemented in the 'Fast Slew' mode of the BIROS attitude control system for in-orbit tests.

\section{Acknowledgments}

The author wishes to thank colleagues at the DLR Institute of Optical Sensor Systems (DLR-OS) in Berlin-Adlershof, for the detailed BIROS satellite data provided; as well as for the preliminary tests performed in the three-axis air-bearing testbed and the opportunity to assist in the first 'Fast Slew' mode in-orbit experiments. Colleagues at the DLR Institute of System Dynamics and Control (DLR-SR) are also acknowledged for their suggestions and discussions leading to improvements in the results obtained during this study. The author also wishes to thank the anonymous reviewers for their valuable suggestions and comments to improve the quality of this paper. 


\section{References}

1) Halle, W., Terzibaschian, T., and Rockwitz, K.-D.: The DLR-BIROSSatellite for Fire-Detection and Technological Experiments, Proc. of the 10th IAA Symposium on Small Satellites for Earth Observation, Berlin, 2015.

2) Halle, W., Terzibaschian, T., Bärwald, W., and Schultz, C.: The DLR Small-Satellite Constellation FireBIRD, Proc. of the 31st International Symposium on Space Technology and Science (ISTS), MatsuyamaEhime, 2017.

3) Briess, K., Bärwald, W., Gill, E., Kayal, H., Montenbruck, O., Montenegro, S., Halle, W., Skrbek, W., Studemund, H., Terzibaschian, T., and Venus, H.: Technology Demonstration by the BIRD-mission, Acta Astronautica, 56 (2005), pp. 57-63.

4) Zhukov, B., Briess, K., Lorenz, E., Oertel, D., and Skrbek, W.: Detection and Analysis of High-temperature Events in the BIRD Mission, Acta Astronautica, 56 (2005), pp. 65-71.

5) Raschke, C., Terzibaschian, T., and Halle, W.: High Agility Demonstration with a New Actuator System by Small Satellite BIROS, Proc. of the 9th Airtec, Frankfurt/Main, 2014.

6) Raschke, C., Terzibaschian, T., and Halle, W.: A New Actuator System for High Agility Demonstration with the Small Satellite BIROS, Proc. of the 10th IAA Symposium on Small Satellites for Earth Observation, Berlin, 2015.

7) Stoltz, S., Raschke, C., and Courtois, K.: RW-90, a Smart Reaction Wheel-Progress from BIRD to TET-1, Proc. of the 8th IAA Symposium on Small Satellites for Earth Observation, Berlin, 2011.

8) Löw, S., Herman, J., Schulze, D., and Raschke, C.: Modes and More; Finding the Right Attitude for TET-1, Proc. of the 12th International Conference on Space Operations (SpaceOps), 2012.

9) Raschke, C., Nicolai, A., Deckert, A., and Stoltz, S.: Development, Test and Operation of the Attitude Control System of the TET-1 Satellite, Proc. of the 29th International Symposium on Space Technology and Science (ISTS), Nagoya-Aichi, 2013.

10) Raschke, C., Terzibaschian, T., Yoon, Z., Stoltz, S., Deckert, A., and Nicolai, A.: The Attitude Control System of the TET-1 Satellite-Inorbit Experiences, Proc. of the 9th IAA Symposium on Small Satellites for Earth Observation, Berlin, 2013.

11) Bilimoria, K. D. and Wie, B.: Time-optimal Reorientation of a Rigid Axisymmetric Spacecraft, Proc. of the AIAA Guidance, Navigation, and Control Conference, New Orleans, LA, 1991.

12) Bilimoria, K. D. and Wie, B.: Time-optimal Three-axis Reorientation of a Rigid Spacecraft, J. Guid. Control Dynam., 16 (1993), pp. 446-452.

13) Bai, X. and Junkins, J. L.: New Results for Time-optimal Three-axis Reorientation of a Rigid Spacecraft, J. Guid. Control Dynam., 32 (2009), pp. 1071-1076.

14) Junkins, J. L. and Turner, J. D.: Optimal Spacecraft Rotational Maneuvers, Elsevier Publishing, New York, 1986.

15) Li, F. and Bainum, P. M.: Numerical Approach for Solving Rigid Spacecraft Minimum Time Attitude Maneuvers, J. Guid. Control Dynam., 13 (1990), pp. 38-45.

16) Byers, R. M. and Vadali, S. R.: Quasi-closed-form Solution to the Time-optimal Rigid Spacecraft Reorientation Problem, J. Guid. Control Dynam., 16 (1993), pp. 453-461.

17) Ross, I. M., Sekhavat, P., Fleming, A., and Gong, Q.: Optimal Feed- back Control: Foundations, Examples, and Experimental Results for a New Approach, J. Guid. Control Dynam., 31 (2008), pp. 307-321.

18) Fleming, A., Sekhavat, P., and Ross, I. M.: Minimum-time Reorientation of a Rigid Body, J. Guid. Control Dynam., 33 (2010), pp. 160-170.

19) Zhou, H., Wang, D., Wu, B., and Poh, E. K.: Time-optimal Reorientation for Rigid Satellite with Reaction Wheels, Int. J. Control, 85 (2012), pp. 1452-1463.

20) Karpenko, M., Bhatt, S., Bedrossian, N., and Ross, I. M.: Flight Implementation of Shortest-time Maneuvers for Imaging Satellites, J. Guid. Control Dynam., 37 (2014), pp. 1069-1079.

21) Lee, T., Leok, M., and McClamroch, N. H.: Time Optimal Attitude Control for a Rigid Body, Proc. of the American Control Conference (ACC), Seattle, WA, 2008, pp. 5210-5215.

22) Lee, T.: Geometric Tracking Control of the Attitude Dynamics of a Rigid Body on SO(3), Proc. of the American Control Conference (ACC), San Francisco, CA, 2011, pp. 1200-1205.

23) Chaturvedi, N. A., Sanyal, A. K., and McClamroch, N. H.: Rigid-body Attitude Control, IEEE Control Syst., 31 (2011), pp. 30-51.

24) Goodarzi, F., Lee, D., and Lee, T.: Geometric Nonlinear PID Control of a Quadrotor UAV on SE(3), Proc. of the 2013 European Control Conference (ECC), Zurich, 2013.

25) Schnepper, K.: MOPS - Trajectory Optimization Package, User's Guide V1.0, DLR-Internal Report, 2014.

26) Joos, H.-D.: A Methodology for Multi-objective Design Assessment and Flight Control Synthesis Tuning, Aerospace Sci. Technol., 3 (1999), pp. 161-176.

27) Joos, H.-D., Bals, J., Looye, G., Schnepper, K., and Varga, A.: A Multi-objective Optimisation-based Software Environment for Control Systems Design, Proc. of the IEEE International Conference on Control Applications and International Symposium on Computer Aided Control Systems Design (CCA/CACSD), Glasgow, 2002.

28) Joos, H.-D.: MOPS - Multi-Objective Parameter Synthesis, User's Guide V6.6, DLR-Internal Report DLR-IB-SR-OP-2016-128, 2016.

29) Matlab Release 14b, The MathWorks Inc., 2014, Natick, Massachusetts, USA.

30) Joos, H.-D.: RCAM Design Challenge Presentation Document: MultiObjective Parameter Synthesis (MOPS), GARTEUR/TP-088-16, 1996.

31) Joos, H.-D., Varga, A., Finsterwalder, R., and Bals, J.: Eine Integrierte Optimierungsbasierte Entwurfsumgebung für Flugregelungsaufgaben, at-Automatisierungstechnik, 47 (1999), pp. 239-248 (in German).

32) Joos, H.-D. and Finsterwalder, R.: Multi-objective Design Assessment and Control Law Synthesis Tuning for Flight Control Development, Proc. of the IEEE International Symposium on Computer Aided Control System Design (CACSD), Hawaii, 1999.

33) Looye, G. and Joos, H.-D.: Design of Autoland Controller Functions with Multi-objective Optimization, Proc. of the AIAA Guidance, Navigation, and Control Conference and Exhibit, Monterey, CA, 2002.

34) Looye, G. and Joos, H.-D.: Design of Autoland Controller Functions with Multiobjective Optimization, J. Guid. Control Dynam., 29 (2006), pp. 475-484.

T. Kida

Associate Editor 